\title{
TRASH CLEANING ROBOT ROVER
}

\section{JIBIN P.S, RESHA REMESH, SURAMYA RAMACHANDRAN \& CHINCHU JOSE}

Electronics and communication department, Sahrdaya college of engineering and technology, Kodakara, India

Received: Jun 08, 2020; Accepted: Jun 28, 2020; Published: Sep 17, 2020; Paper Id.: IJMPERDJUN20201309

\section{I.INTRODUCTION}

The improper waste disposal has a serious impact on a wide range of areas. The garbage thrown all around our provinces and associated areas can cause much difficulty to the public endurance and the cleaning also comes with much difficulty for all of us manual humans. The various layers of environment have been subjected to high degrees of pollution which masks the health of people with contagious as well as other serious diseases. Where all these problems are common in India, so the vast quantities of solid waste remain uncollected in streets, empty plots of lands etc. The time constraint required for many tedious tasks can be limited and reduced to large scale as well as the amount of work. The trash cleaning robot rover that we put forward is a robot that can plunge itself to the geometrically arising cleaning based deeds that is to be performed and induced on the individual is also reduced on geometric level. The robot chassis that we put forward is predominant as of a perfect sleek design that an ideal cleaning machine should possess enough to the chores that are practically difficult and time consuming for any of us. In short there is an additional tool attached to the rover who is quite similar to a dust pan in our common words, this serves the purpose of collecting the waste from all along our surroundings. The chosen idea of the dust pan provides the ease and simplicity to our design adding to the efficiency by serving to minimum risk in time handling and complexity. Many of the existing ideas that we came across are more complex in design and its seen that although a lot of emphasis has been excreted on these designs the outcome has not met the standards that are expected out of it, so the idea of having a simple design that can be understood easily and used by households and also by people who have minimum knowledge on technology is also really important . A design should not only be perfect but also meet the standards in such a way it understood and accepted by common people. In the current scenario of pollution and various health issues arising due to this discipline less disposal of waste has posed a heavy threat on all of us, all over the world. Its high time we understand the importance of this and the kind of harm it can cause to all of us. The human nature of neglecting the act of keeping the surroundings clean is also adding to the problem. Although with the high pace of technological developments that has been happening, the 
availability of laborer still remains a constant. The effect of such an arising global issue explains the need and implementation of the idea by itself .The rover that we put forward is perfectly compatible on both perfect for parks, beaches ,hospitals, educational institutions like schools, colleges etc and practically anywhere where manual interference is technically difficult. the exact purpose of collecting waste with a

\section{HARDWARE CONCEPT}

Due to the high cost of the normal driver circuit, we by ourselves have come across an idea that we mapped into the construction of our own motor driver circuit. A motor generally requires a current of $9 \mathrm{~A}$, also it required $12 \mathrm{v}$ for its proper functioning. As this by itself requires a wholesome amount of money, we reconstructed the idea into budgeted one. The motor driver circuit consists of two relays and also the same amount of mosfets. Relays are directed to change the polarity. The mosfets are used here are namely NF6010 and hey spectacularly handle the service of voltage regulation. The regulation applied as of between range of 0-5v. Since we obtain the output from Arduino as this specified range, the above selected NF6010 mosfet does the purpose in an absolute perfect manner

The positive terminal of battery is directly connected to the common part of the relay and the negative terminal well connected to the source of the mosfet. We obtain the output from the drain. As we are using a DPDT (double pole double throw) relay, we connect the same to the next common part of the relay. Triggering of the relay is done using a microcontroller, polarity changes accordingly and output is fetched and received by motor. This is the basic working of motor driver circuit.

\section{PURPOSED METHOD}

\section{A. Camera Capture the Object}

Here in order to the satisfy the purpose of image processing, we used a software tool called OpenCV which can be in consolidate defined as an open source computer vision and machine learning software library. It includes a library of programming functions mainly aimed at real-time computer vision. A USB camera of 385 mega pixels is been used. A deep learning methodology is induced and the predominance of object detection on video slams can be seen. Object detection can be explained as a computer vision technique for locating instances of objects in images or videos. We use lmutils package that makes the hectic and heavy tasks of image processing sound and look much easier and better. Next, we look up on the code and active initializing of class labels and some random colors for additional information and subsequent details. This is rapidly followed by the loading of our content and setting up of a suitable environment that enables the successful completion of our work. The video stream from the camera we used here is initialized, for this we start the process of video streaming and wait for the camera to warm up. The GPS counter is then put to a start. Then we will have to loop the outer energy frames followed by the reading of frames. Subsequently we resize the frames and convert it to blob with module. We set blob on input to our neural network and followed providing the inputs after which we get the the preceded outputs as detections. The detected objects in inputs in input frame should check for confidence values and for easy user interface decide whether to draw a box, label surrounding the object. 


\section{BLOCK DIAGRAM}

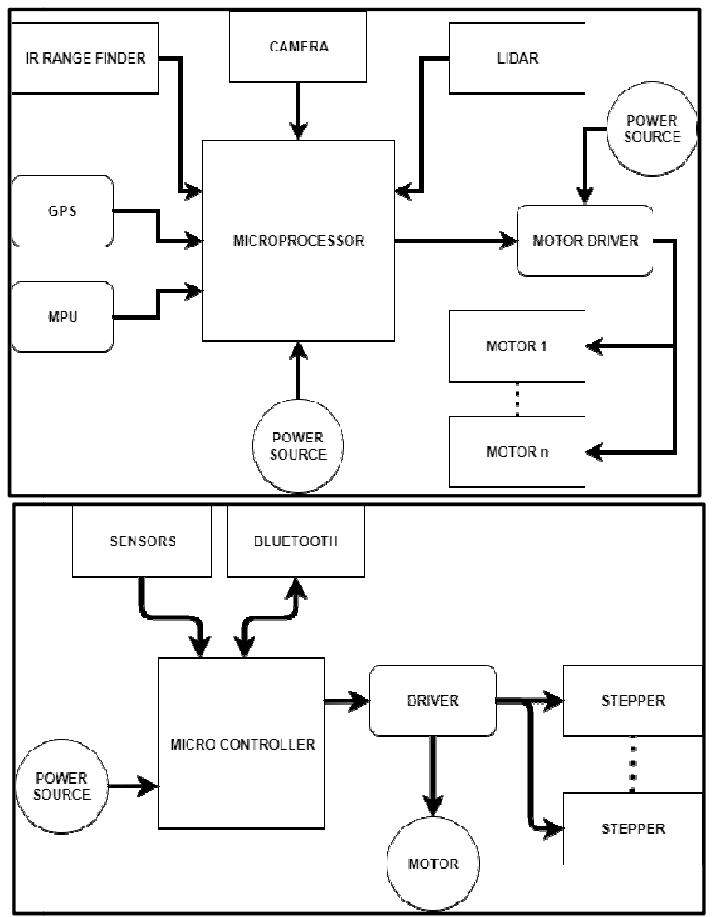

\section{B. Formation of the Mechnical Structure}

A mechanical structure is quite essential element in a design; basically it is the backbone that holds all the essentials right from the scratch. The mechanical design we incorporated here consists of a primary base made of aluminum foil having a suitable chosen dimension of $50 * 30 \mathrm{~cm}$. Then by a simple procedure of riveting and drilling we attain all our positions and joints in position intact. Next step is mounting a structure which is identical to the shape of a box, the box not only serves the purpose of containing the circuits that centralize the rover, pi, Arduino, but also provides a completion of aesthesis to the design formulated and provides the room to hold a slantingly positioned structure that is structurally similar to a dust pan. The selection of a dust pan similar structure not only is similar to one, it simultaneously does work and faultlessly provides the objective of it too. This dust pan similar structure that provides the primary purpose of collection of waste, materials with minimum chances of any mis functionality is efficiently controlled by the use of dc motors. The idea of the dust pan has evolved from the process of equally portioning a conventional dustbin that all of us are familiar in acquainting within our day to day lives. Enough provisions have been made incorporating all the software and relatable codes to the above mentioned dust pan structure to automatically perform the process of collection the waste and subsequently disposes the wastes after the bin gets filled.

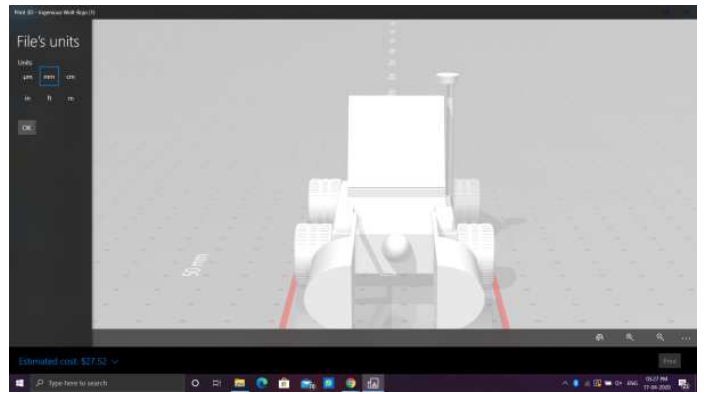

Figure a: 3D view of robot rover drawn using TinkerCAD 


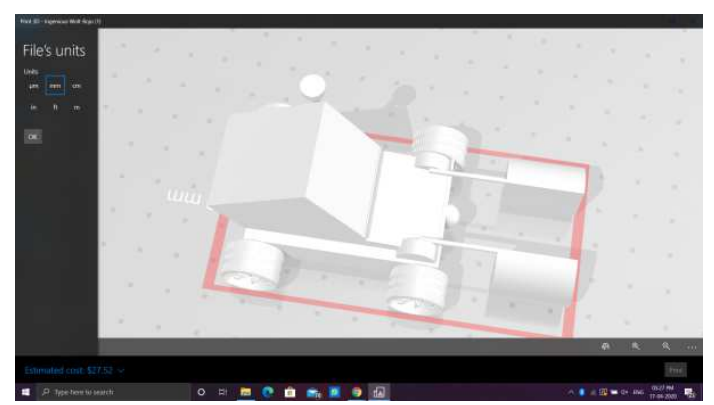

Figure b: Top view of the structure

\section{Main Working}

The robot rover moves inside a particular boundary of specified coordinates. Using lidar and other type of sensors the obstacle avoidance can be removed. Lidar is sensor which we placed top on the rover, which delivers high performance in 3D sensing. A typical lidar sensor emits pulsed light waves into the surrounding environment. Once the object is detected the object boundary is marked. There is a fixed position in the frame where the arm of the robot could grab the object. So once the object is detected its X,Y position in the frame is read. Then robot performs required motion to bring the object in the robotic arms grabbing position. Once the object is in the grabbing position the object is grabbed and is placed on the basket on the rover.

\section{CONCLUSIONS}

The problem of time consumption as well as the tedious deposition of work on laborers or people associated with the cleaning based chores is reduced on a much greater level the arising problem of pollution due to improper waste disposal due to the carelessness of people can also be reduced to a much greater level.

\section{V.RESULT AND DISCUSSIONS}

We successfully implemented a robot which is helping for to picking up the trashes from nearest distance. And also the robot itself dumped the trashes into the assigned area. LiDAR is an airborne sensing technology which makes data collection fast and comes with extremely high accuracy as a result of the positional advantage. It is a technology that collects very huge datasets that require high level of analysis and interpretation. For this reason, it may take a lot of time to analyze the data.

\section{ACKNOWLEDGEMENTS}

It gives us immense pleasure to express our deepest sense of gratitude and sincere thanks to our highly respected and esteemed guide CHINCHU JOSE, for his valuable guidance, encouragement and help for completing this work. We would like to express our sincere thanks to CHINCHU JOSE, forgiving us this opportunity to undertake this project. We would like to thank our executive director, REV.FR. GEORGE PARAMEN, principal Dr.NIXON KURUVILA for their support and encouragement. We also wish to express our gratitude to Dr.VISHNU RAJAN HOD (Electronics and Communication Engineering) for giving us constant inspiration. We are also grateful to all our teachers for their constant support and guidance. We also wish to express our indebtedness to our parents as well as our family member whose blessings and support always helped us to face the challenges ahead. At the end, we would like to express our sincere thanks to all our friends and others who have helped us directly or indirectly during this project work. 


\section{REFERANCES}

1. Y. Dedeoglu, Moving object Detection, Tracking and Classification for Smart Video Surveillance, Bilkent University,2004

2. https://www.pyimagesearch.com/2018/09/26/install-opencv-4-on-your-raspberry-pi/

3. K. H. Sedighi, T. W. Manikas, K. Ashenayi, and R. L. Wainwright, international Journal of Robotics and Automation, vol. 24, no. 6,pp. 367-373,2009.

4. https://create.arduino.cc/projecthub/Aritro/getting-started-with-imu-6-dof-motion-sensor-96e066

5. http://www.acuitylaser.com/products/category/long-range-sensors/

6. Hesham Alsahafi and Majed Almaleky, "Design and Implementation of Metallic Waste Collection Robot,” ASEE 2014 Zone I Conference, April 3-5, 2014, University of Bridgeport, Bridgpeort, CT, USA., p. 6.

7. G. Maarten Bonnema $a^{*}$, "System design of a litter collecting robot," New Challenges in Systems Engineering and Architecting Conference on Systems Engineering Research (CSER) 2012 - St. Louis, MO, p. 6.

8. Xiaoli Wang, “Object extraction technique based on OPENCV and VC++ ,"in SILICON VALLEY, 2010,pp.164-165. 

